\title{
Identification of a novel iron regulated basic helix-loop-helix protein involved in Fe homeostasis in Oryza sativa
}

\author{
Luqing Zheng, ${ }^{1,2}$, Yinghui Ying ${ }^{1,2}$, Lu Wang ${ }^{1,2}$, Fang Wang ${ }^{1}$, James Whelan ${ }^{1,3}$, Huixia Shou ${ }^{1,2^{*}}$
}

\begin{abstract}
Background: Iron (Fe) is the most limiting micronutrient element for crop production in alkaline soils. A number of transcription factors involved in regulating Fe uptake from soil and transport in plants have been identified. Analysis of transcriptome data from Oryza sativa grown under limiting Fe conditions reveals that transcript abundances of several genes encoding transcription factors are altered by Fe availability. These transcription factors are putative regulators of Fe deficiency responses.

Results: Transcript abundance of one nuclear located basic helix-loop-helix family transcription factor, OsIRO3, is up-regulated from 25- to 90-fold under Fe deficiency in both root and shoot respectively. The expression of OsIRO3 is specifically induced by Fe deficiency, and not by other micronutrient deficiencies. Transgenic rice plants overexpressing OsIRO3 were hypersensitive to Fe deficiency, indicating that the Fe deficiency response was compromised. Furthermore, the Fe concentration in shoots of transgenic rice plants over-expressing Os/RO3 was less than that in wild-type plants. Analysis of the transcript abundances of genes normally induced by Fe deficiency in OsIRO3 over-expressing plants indicated their induction was markedly suppressed.

Conclusion: A novel Fe regulated bHLH transcription factor (OsIRO3) that plays an important role for Fe homeostasis in rice was identified. The inhibitory effect of OsIRO3 over-expression on Fe deficiency response gene expression combined with hypersensitivity of OsIRO3 over-expression lines to low Fe suggest that OsIRO3 is a negative regulator of the Fe deficiency response in rice.
\end{abstract}

\section{Background}

Iron $(\mathrm{Fe})$ is an essential micronutrient for plant growth and production. This is due to the fact that it is an essential co-factor in a variety of enzymes that play critical roles in photosynthesis, respiration and nitrogen fixation [1]. Although Fe is the second most abundant metal element in the earth crust, its bio-availability is limited, especially in alkaline soils where Fe largely exists as insoluble hydroxides or oxides [2]. While the optimal Fe concentration for plant growth is in the range of $10^{-9}$ to $10^{-4} \mathrm{M}$, the bio-available Fe in most soils is approximately $10^{-17} \mathrm{M}[2,3]$. Plants have two distinct uptake strategies to increase the efficiency of Fe uptake from soil [4]. The reduction strategy employed by non-grass

\footnotetext{
* Correspondence: huixia@zju.edu.cn

'Joint Research Laboratory in Genomics and Nutriomics, College of Life

Sciences, Zhejiang University, Hangzhou 310058, P R China

Full list of author information is available at the end of the article
}

plant species uses a Fe deficiency induced reductase to convert insoluble $\mathrm{Fe}(\mathrm{III})$ to $\mathrm{Fe}(\mathrm{II})$, the latter being transported into plant cells by the $\mathrm{Fe}(\mathrm{II})$ transporter IRT1 [4-6]. In contrast, grass species use a chelating strategy to obtain Fe from soil. The chelating strategy consists of Fe deficiency induced biosynthesis and secretion of phytosiderophore(s) and cognate high affinity transporters, Fe(III)-phytosiderophore Yellow Stripe Transporter, ZmYS1, HvYS1 and OsYSL15 [7-9].

Many of the components involved in Fe uptake for both of the strategies outlined above have been identified at a molecular level. In Arabidopsis $\mathrm{H}^{+}$-ATPase 2 (AHA2) that mediates acidification of the rhizosphere [10], a ferric reductase FRO2 [6] and a ferrous Fe transporter IRT1 [5], represent the three key components required for a strategy I Fe uptake system. Identification of the molecular components involved in strategy II Fe uptake system has focused on the biosynthesis of the Fe 
(III)-chelator, phytosiderophore (PS) [11]. Key genes in the PS biosynthesis include nicotianamine synthase gene OsNAS1/2/3 [12], nicotianamine aminotransferase gene OsNAAT1 [13], and the cognate transporter YellowStripe 1 [7].

Many of the genes involved in Fe uptake via strategy I or II display a distinct Fe deficiency induced expression pattern [14]. Transcriptome studies have shown that large scale alterations of transcript abundances is a common feature of Fe deficiency and thus plays a key role in the Fe deficiency response [15-17]. Alterations in transcript abundances of genes encoding transcription factors are of particular interest in these studies due to their potential role in regulating the Fe deficiency response. The bHLH transcription factor FER, a regulator of iron uptake, was first identified from the analysis of the tomato fer mutant [18]. The fer mutant failed to activate a Strategy I Fe uptake pathway under Fe deficient conditions. Studies have also shown that either FER or its Arabidopsis ortholog, FER-like transcription factor, FIT1, is required for the strategy I responses [19]. Subsequent studies have identified a family of bHLH transcription factors in Arabidopsis (AtbHLH 38/ $39 / 100 / 101)$ that interact with FIT1 [20,21]. A number of transcription factors involved in regulating the $\mathrm{Fe}$ deficiency response in Oryza sativa (rice) have also been identified. The Fe-regulated bHLH transcription factor, OsIRO2, shares high similarity with the Arabidopsis family of bHLH transcription factors and positively regulates the expression of strategy II pathway genes, including OsNAS1/2/3, OsNAAT1, deoxymugineic acid synthase gene OsDMAS1 and a YS-like gene OsYSL15 [22]. Two other transcription factors, Fe-deficiencyresponsive factors, IDEF1 and IDEF2, bind the Fe-deficiency-responsive elements 1 and 2 (IDE1 and IDE2) and positively regulate a large number of $\mathrm{Fe}$ responsive genes [23-25]. As $I D E F 1$ and IDEF2 are constitutively expressed in roots and shoots in rice, other regulatory factors which may only be expressed under Fe limiting conditions may be involved in regulating the response to Fe deficiency.

A previous study identified transcripts of several genes encoding transcription factors that changed in abundance with Fe deficiency [17]. In this study, the function of one of the transcription factors, a bHLH transcription factor (TF) family protein, named OsIRO3 (OsIRbHLH2 in previous paper, LOC_Os03g26210) was investigated. Our analysis suggests that OsIRO3 acts as a negative regulator of the Fe deficiency response.

\section{Results}

\section{OsIRO3 is specifically induced by Fe deficiency}

Previous studies in our laboratory identified several transcription factors whose expression was induced by
Fe deficiency in rice [17]. One gene encoding a bHLH transcription factor, OsIRO3, is highly induced by Fe deficiency in both roots and shoots [17]. To determine whether the induction of OsIRO3 is specific to Fe deficiency or whether this induction could be observed under deficiency of other minerals, the transcript abundance of OsIRO3 was determined from plants grown under a variety of different mineral element deficient conditions. The transcript abundance of OsIRO3 was markedly induced by Fe deficiency in both roots and shoots, by 25 - and 90 -fold respectively (Figure 1A). Deficiency of several other minerals, including copper and zinc had no effect of the induction of OsIRO3 in roots or shoots (Figure 1A), indicating that the induction of OsIRO3 was specific to Fe deficient conditions.

The time-course expression pattern of the OsIRO3 and the other two known transcription factors, OsIRO2 and IDEF1, in response to Fe deficiency was investigated. Consistent with previous studies $[15,22,24], I D E F 1$ was constitutively expressed in both roots and shoots (Figure 1B). In contrast OsIRO2 and OsIRO3 were significantly up-regulated by Fe deficiency, with a 10 -fold induction or greater evident after 3 days and continued induction up to 8 days (Figure 1B). The induction of OsIRO3 was less in terms of magnitude in shoots, OsIRO3 was induced 20 -fold compared to OsIRO2 being induced almost 40 -fold after 6 days (Figure 1B shoot). In roots OsIRO3 induction lagged behind that of OsIRO2 both in time and magnitude, OsIRO2 was already induced 50 -fold after 1 day compared to OsIRO3 for which a 10 -fold induction was observed only after 3 days (Figure 1B root).

\section{Subcellular localization of $\mathrm{OsIRO3}$}

To determine the subcellular localization of OsIRO3 protein, the full length cDNA of OsIRO3 was cloned in frame to the $3^{\prime}$ end of the $S G F P$. The expression of the fusion protein was driven by the $35 \mathrm{~S}$ CaMV promoter. Targeting ability was tested in onion epidermal cells using microprojectile bombardment along with the vector control which contains $s G F P$ alone. Results showed that the OsIRO3::sGFP fusion protein was targeted to nuclei (Figure 2A to 2C). The control sGFP was found throughout the cell, in nuclei and the cytosol (Figure 2D to $2 \mathrm{~F}$ ), indicating that OsIRO3 has specific nuclear targeting ability.

\section{Phylogenetic analysis of OsIRO3}

There are 158 and 173 predicted members of the bHLH-domain containing transcription factors in Arabidopsis and rice respectively [26,27]. To investigate the relationship between OsIRO3 and previously characterized bHLH transcription factors regulating the $\mathrm{Fe}$ 


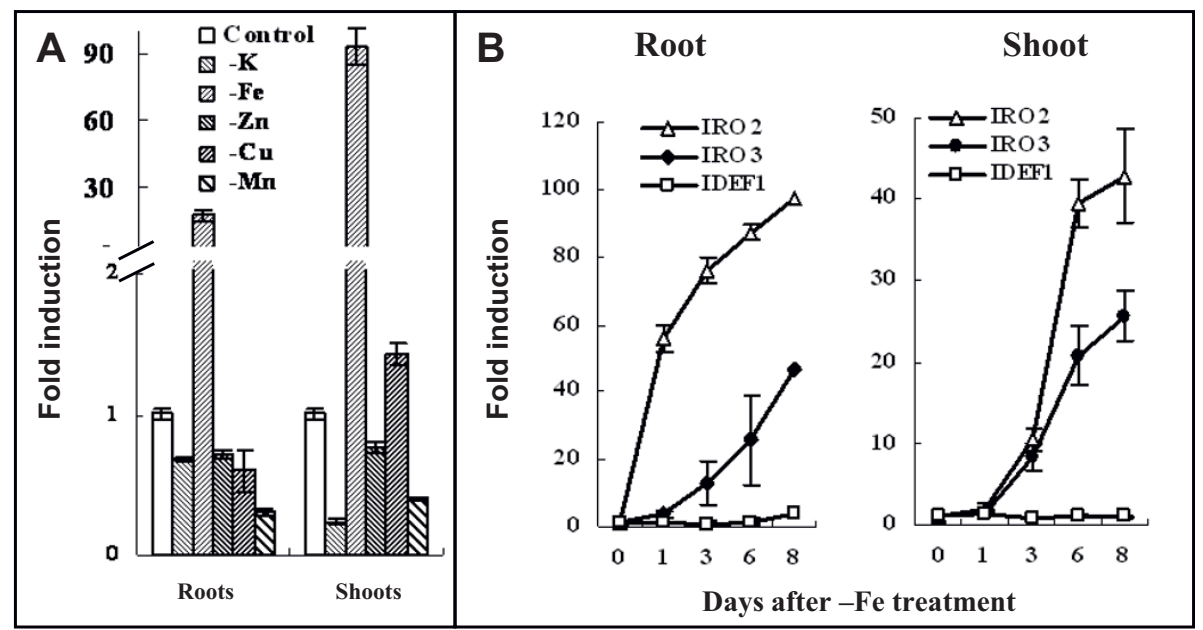

Figure 1 Expression analysis of OsIRO3. Three week old rice seedlings were transferred to solution culture with different metal element deprivation (-K, - Fe, $-\mathrm{Zn},-\mathrm{Cu},-\mathrm{Mn})$ for eight days. Root and leaf samples were separately analyzed and the control expression level was normalized to 1 in both root and leaves. (A) Expression of OsIRO3 under different nutrient deficiency conditions. (B) Time-course transcript abundance of OsIRO3, OsIRO2 and IDEF1 in response to Fe deficiency.

deficient response in Arabidopsis and rice, a phylogenetic analysis using amino acid sequences of OsIRO3 and the previously characterized bHLH transcription factors tomato FER [18], Arabidopsis FIT1 [19], AtbHLH 38, 39, 100 and 101 [20] was performed (Amino acid sequences were listed in Additional file 1). As expected, FER and its Arabidopsis ortholog, FIT1 were classified into the same Clade (Figure 3, Clade I). OsIRO2 branches in the same clade with the
Arabidopsis bHLH family proteins 38/39/100/101 (Figure 3, Clade II). OsIRO3 branches into Clade III (Figure 3). AtbHLH105 (ILR3) which was reported to modulate metal homeostasis and response to auxin was also found in this clade [28]. The protein sequence of Arabidopsis ILR3 is only $20.9 \%$ identical to OsIRO3. Also, OsbHLH62 (OsbHLH1), that branches closest to OsIRO3 (Figure 3), has been previously reported to be induced by cold stress [29].

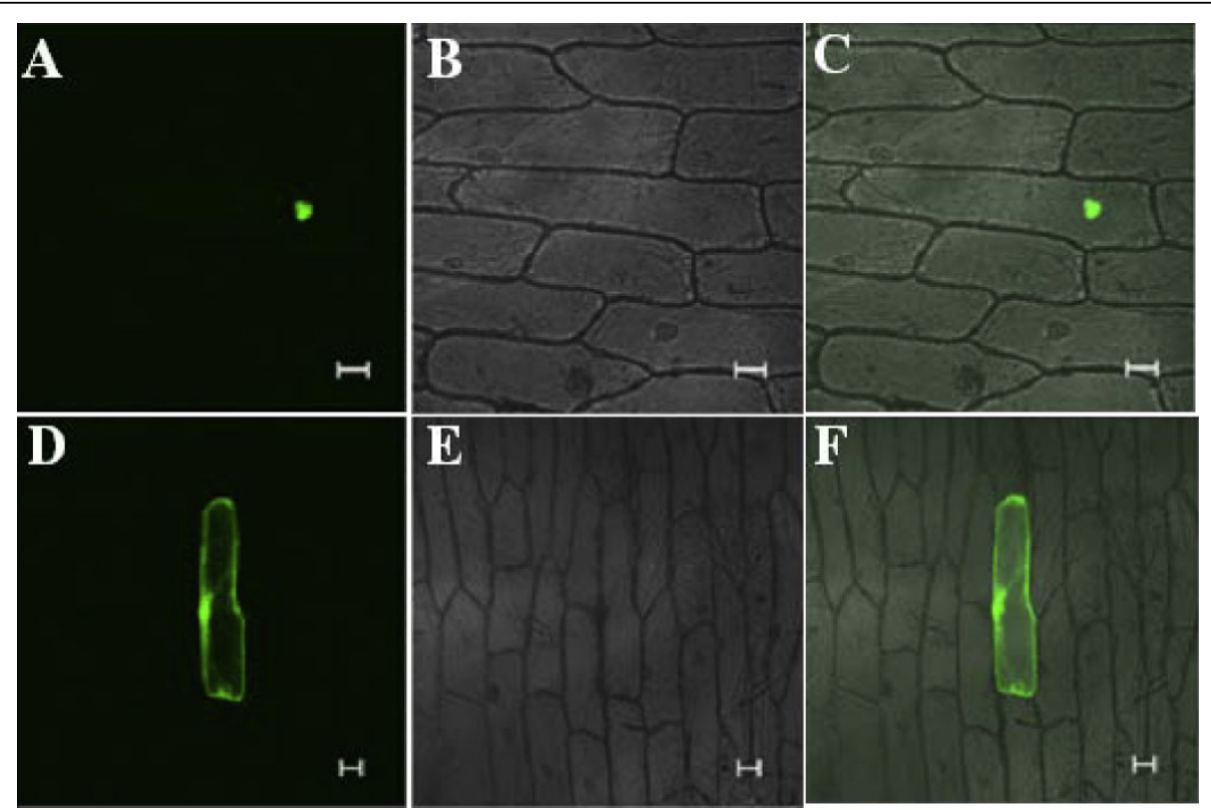

Figure 2 Subcellular localization of OsIRO3. Confocal images of onion epidermis cells expressing OsIRO3-sGFP (A, B, C) and sGFP (D, E, F). Images were taken under GFP channel ( $A$ and $D)$, under transmitted light ( $B$ and $E)$, and their merged images $(C$ and $F)$. Bar $=50 \mu m$. 


\section{Clade III}

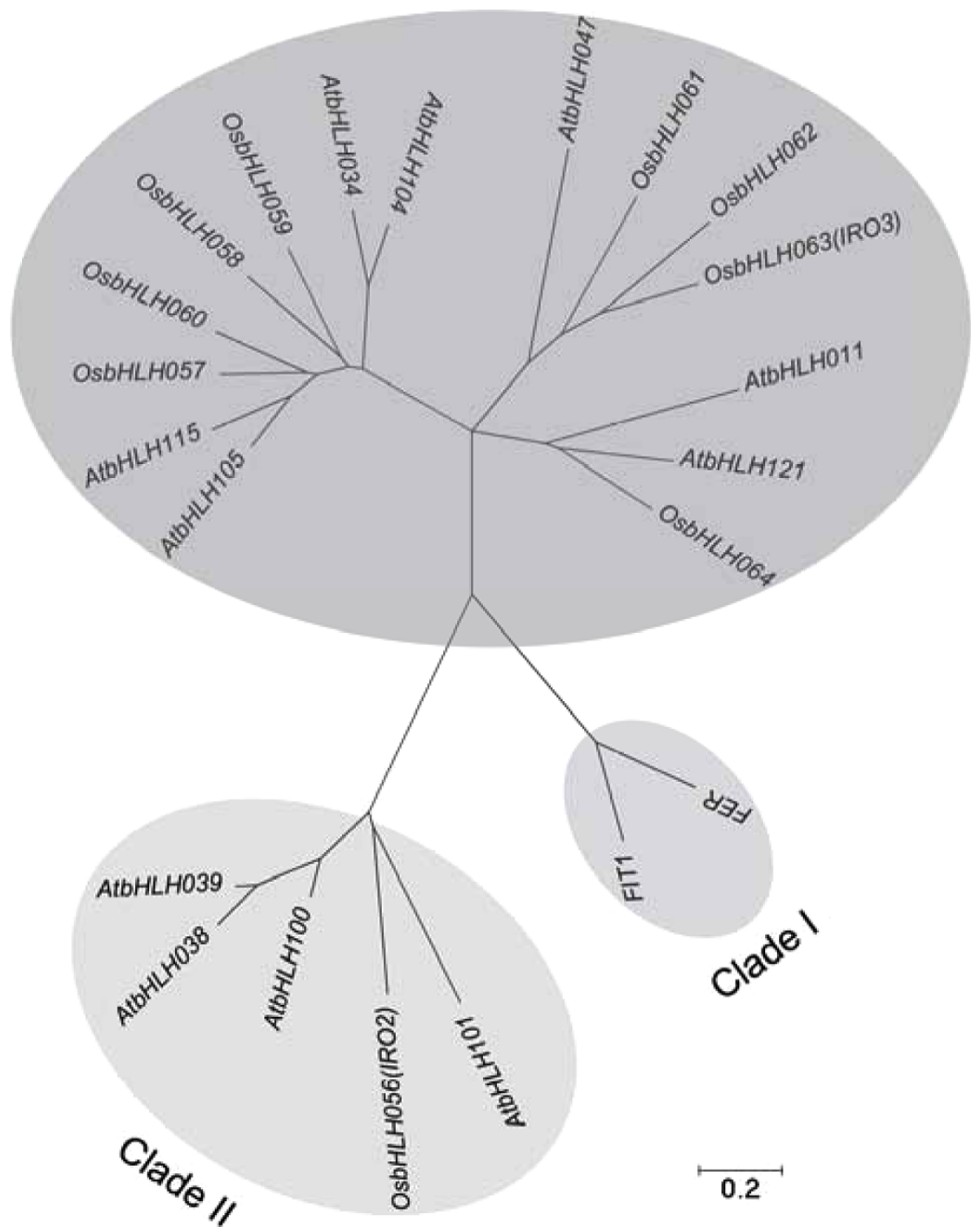

Figure 3 Phylogenetic tree of OsIRO3-like bHLHs with known bHLH transcription factors that function in the Fe deficient response bHLH transcription factors from Arabidopsis and rice were subjected to the neighbor-joining algorithm phylogenetic tree construction using MEGA software version 4.1 [37] with default settings. The amino acid sequences used to generate this phylogenetic analysis are listed in Additional file 1.

\section{Over-expressing OsIRO3 results in hypersensitivity to Fe deficiency}

OsIRO3 was over-expressed (OE) in rice to investigate the role it played in Fe homeostasis. Two independent OE transgenic lines with significantly altered expression level of OsIRO3 (Figure 4A) were studied in detail. To examine if alteration of OsIRO3 expression in transgenic lines influences tolerance to low Fe conditions, three week old $\mathrm{OE}$ and wild-type rice seedlings were transferred into solution culture containing high $(100 \mu \mathrm{M})$, moderate $(10 \mu \mathrm{M})$, or low $(1 \mu \mathrm{M})$ levels of Fe for 10 days. Under Fe sufficient conditions $(100 \mu \mathrm{M})$, the transgenic seedlings overexpressing OsIRO3 had significantly shorter shoots compared to the wild-type counterparts(Figure 4C, Additional file 2), although the color was normal with no evidence of chlorosis that would suggest Fe deficiency (Figure 4B, Additional file 2). SPAD values and $\mathrm{Fe}$ concentrations from these plants also 


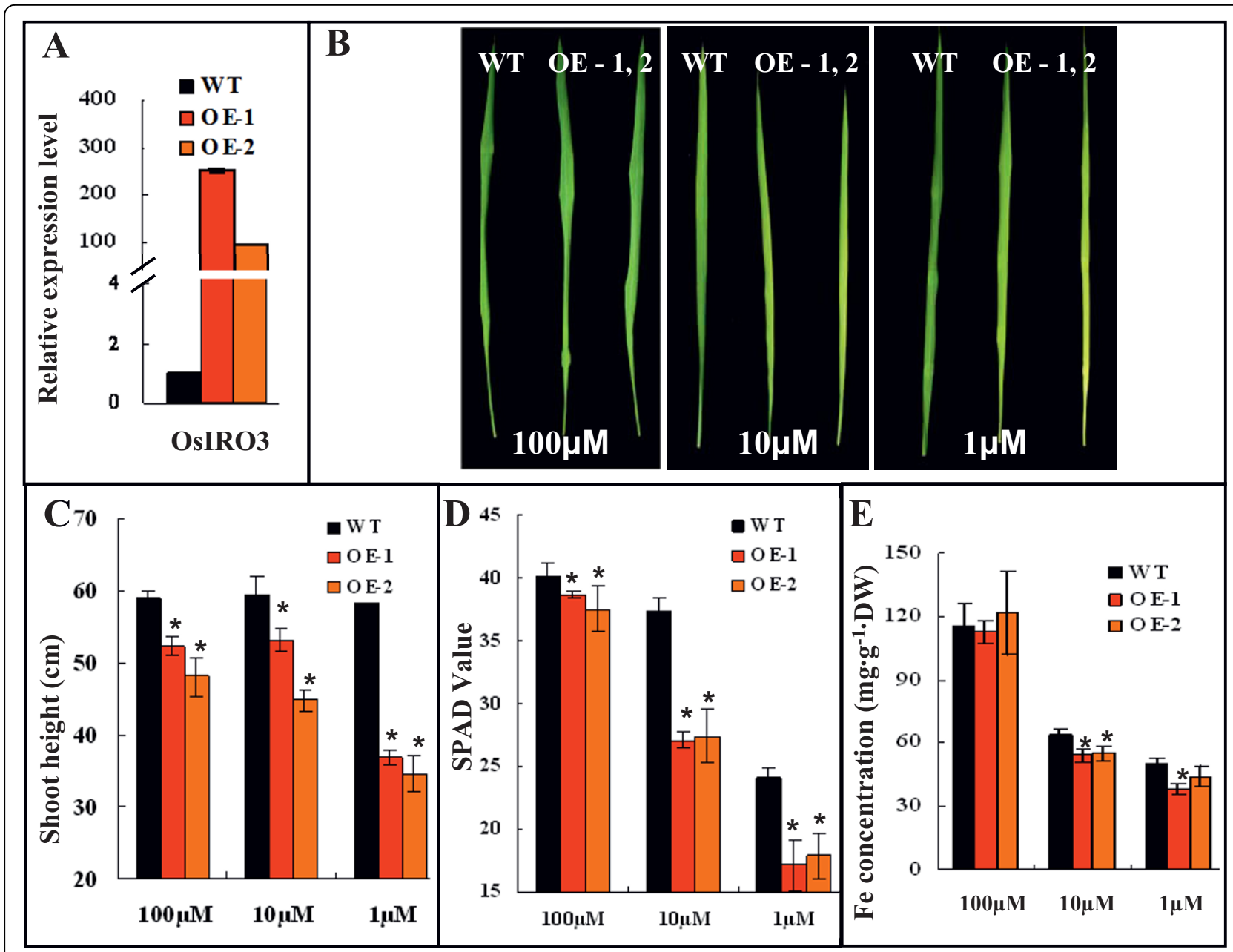

Figure 4 Growth performance and transcript abundance of OsIRO3 in over-expressing (OE) lines. Three week old transgenic and vector only transformed rice plants, the latter referred to as wild-type, were transferred to solution culture containing 100, 10, or $1 \mu \mathrm{M}$ EDTA-Fe for 10 days. (A) Expression levels of OsIRO3 in OE plants grown in normal solution culture compared to wild-type. The expression level of wild-type was normalized to one. (B) The youngest leaf of OsIRO3-OE and wild-type plants in solution culture with $100 \mu \mathrm{M}, 10 \mu \mathrm{M}$, or $1 \mu \mathrm{M}$ Fe-EDTA. (C) Shoot height of OsIRO3-OE and wild-type under the various Fe conditions. (D) SPAD values of newly expanded leaves of OsIRO3-OE and wild-type plants. (E) Shoot Fe concentrations of OSIRO3-OE lines and wild-type.

confirm values very close to wild type, although SPAD values were slightly but significantly lower (Figure 4D and 4E). Under moderate Fe supply $(10 \mu \mathrm{M})$, the two OE lines displayed a chlorotic phenotype in the newly developed leaves compared to the wild-type plants (Figure 4B, Additional file 2). The SPAD values of the youngest leaves in both OE lines under $10 \mu \mathrm{M}$ Fe were reduced to $\sim 65 \%$ compared to the wild-type control (Figure 4D). At $1 \mu \mathrm{M}$ Fe supply, all plants displayed chlorotic symptoms (Figure 4B, Additional file 2). Both OE lines had significantly reduced SPAD values and shoot height compared to wild-type (Figure 4C and 4D, Additional file 2). The Fe concentration of shoots in both OE lines was significantly reduced compared to wild-type under both medium and low levels of Fe supply (Figure 4E).
OsIRO3 suppresses the induction of $\mathrm{Fe}$ responsive genes by Fe deficiency

The expression patterns of previously characterized $\mathrm{Fe}$ deficiency responsive genes, OsNAS1, OsNAS2, OsIRO2, OsIRT1, OsYSL15, and OsNramp1 [5,6,8,12,30] were investigated under different Fe levels. As expected, all of these genes were markedly induced by Fe deficiency (Figure 5). OsNAS1 and OsNAS2 were very sensitive to Fe availability, displaying the highest induction levels under Fe deficiency conditions (Figure 5). However, in the two OE OsIRO3 transgenic lines, the induction of transcript abundance for these genes, with the exception of OsIRT1 in roots, was significantly suppressed under all three $\mathrm{Fe}$ supply conditions (Figure 6A-C). The expression of OsIRT1 was only significantly 


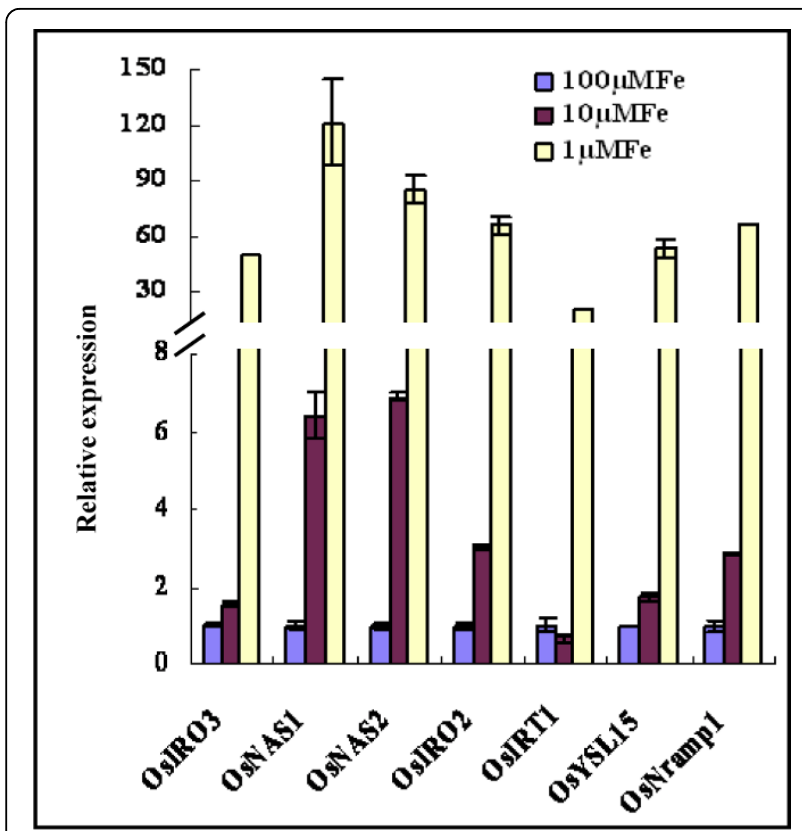

Figure 5 Expression patterns of Fe deficiency response genes in wild type roots grown under different Fe concentrations. Three week old wild-type rice plants were subjected to solution culture containing 100, 10, or $1 \mu \mathrm{M}$ EDTA-Fe for 10 days. Root RNA was prepared for quantitative RT-PCR analysis. For each gene transcript abundance measured under $100 \mu \mathrm{M}$ EDTA-Fe was normalized to one with other values shown as relative expression.

down-regulated by both OE lines under low Fe supply condition (Figure 6C).

\section{Discussion}

Several lines of evidence indicate an important regulatory role for OsIRO3 in response to Fe deficiency in rice. Firstly, it is specifically induced by Fe deficiency but not by other metal depletion. Secondly, OsIRO3-OE lines are more sensitive to low Fe availability than wildtype. Thirdly, the induction of Fe deficiency responsive genes that play pivotal roles in Fe uptake are suppressed in OsIRO3-OE lines. Together these data imply that OsIRO3 is a negative regulator of Fe uptake in rice.

To date, a variety of bHLH transcription factors have been shown to play important roles in regulating $\mathrm{Fe}$ uptake and homeostasis $[15,18,20-22]$. OsIRO3 is not phylogenetically related to other known bHLH transcription factors involved in the regulation of Fe uptake, such as OsIRO2 and IDEF1 [15,24]. Thus, it is a novel Fe-responsive transcription factor. Over-expression of OsIRO2 and IDEF1 increased the expression of Fe uptake related gene transcripts and these plants also showed an enhanced tolerance to Fe deficiency. Thus, they are positive regulators of downstream Fe responsive genes [24]. In contrast, this study revealed that over-expression of OsIRO3 results in hypersensitivity to Fe deficiency, due to the suppression of

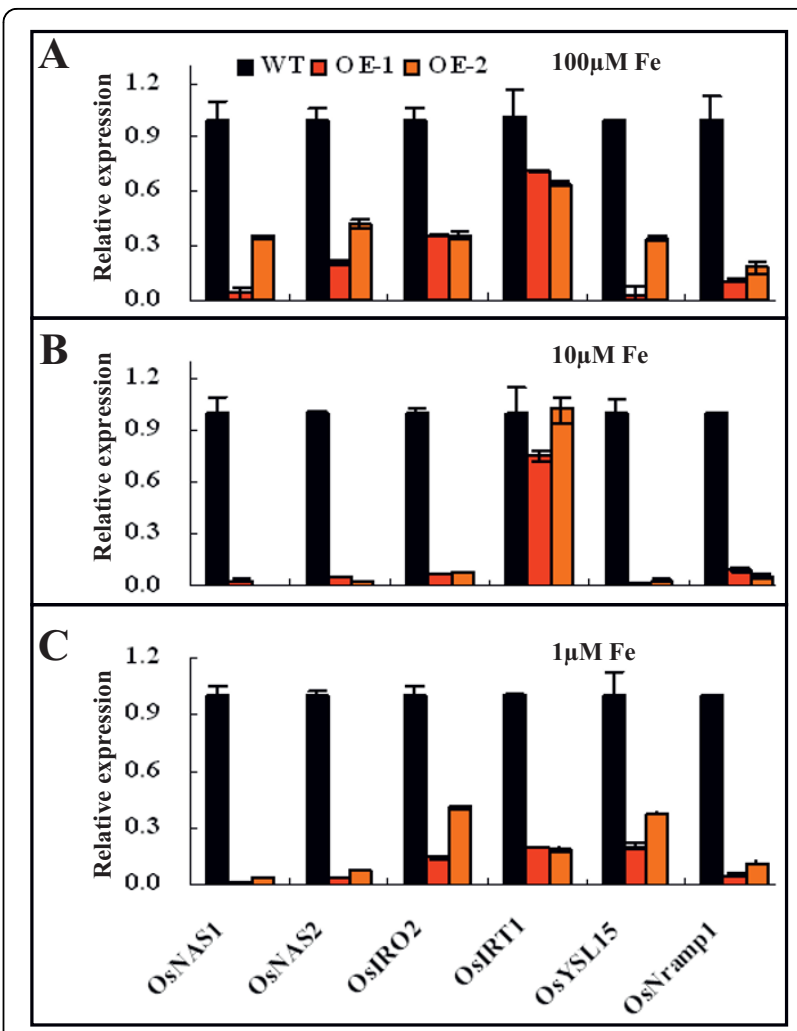

Figure 6 Expression patterns of Fe deficiency response genes in roots of OsIRO3-OE and wild-type plants under $100 \mu \mathrm{M}(\mathrm{A})$, $10 \mu \mathrm{M}(\mathrm{B})$, or $1 \mu \mathrm{M}$ Fe-EDTA (C) conditions. Plant growth

conditions are described in detail in material and method. For each gene transcript abundance measured for wild type plants grown under $100 \mu \mathrm{M}$ EDTA-Fe was normalized to one with other values shown as relative expression.

induction of Fe uptake related genes, suggesting it is a negative regulator. Fe deficiency induces the activation of the methionine cycle and biosynthesis of PS in strategy II plants $[14,17]$, a process which is nutrient and energy demanding. Over-expression of NA synthase alone, an enzyme catalyzing a rate limiting step of NA and PS synthesis resulted in significantly reduced growth [31,32]. In addition, excessive Fe acts as a catalyst for the formation of free radicals that are potent oxidizing agents which can damage many important biological molecules in cells [2]. Thus, although the Fe deficiency response results in increased Fe uptake it also results in a growth penalty. Therefore negative regulators are required to balance the induction of Fe uptake components to reduce the negative effect on plant growth that results from excessive Fe and/ or as a result of the induction of the components due to Fe deficiency. The fact that the induction of OsIRO3 is slightly later in shoots and at a lower level in roots compared to the positive regulator OsIRO2 (Figure 1B) suggests that OsIRO3 may play a role as part of a negative feed back loop on the Fe deficiency response. 
The negative effect of OsIRO3 occurs via either a direct or indirect mechanism. As OsIRO3 induction lags that of OsIRO2, it may be regulated by OsIRO2 to form a negative feedback loop. In fact, there is a putative OsIRO2 binding cis-element in the promoter region of OsIRO3 (Additional file 3). bHLH transcription factors often function in homo- and/or hetero-dimerized forms $[33,34]$. OsIRO3 and OsIRO2 and/or IDEF1 regulate similar downstream targets, albeit in opposite manners. These transcription factors might interact with each other and/or bind to the same cis-acting regulatory elements in the promoter region of target genes. Analysis of the expression of OsIRO3 and IDEF1 in different microarray data sets showed that these two genes are expressed antagonistically (Additional file 4). There are a number of predicted proteins in Arabidopsis and rice with high sequence similarity to OsIRO3 (Figure 2). The closest orthologue of OsIRO3 gene in Arabidopsis, AtbHLH047, is also induced by Fe deficiency (unpublished data). Whether AtbHLH047 plays a similar role in $\mathrm{Fe}$ homeostasis in Arabidopsis needs to be investigated.

\section{Conclusion}

This study reveals the regulatory role of a bHLH protein, OsIRO3 in Fe homeostasis in rice. OsIRO3 is specifically induced by Fe deficiency, suggesting it functions in Fe deficiency responses. The nuclear localization of OsIRO3 supports the hypothesis that OsIRO3 acts as a transcription factor. The hypersensitivity to low Fe supply of the OsIRO3-OE and the inhibitory effect of OsIRO3-OE on the induction of Fe deficiency response genes suggest OsIRO3 functions as a negative regulator in Fe deficiency responses. The identification of the new important regulator adds to a growing understanding of the network of fine control of Fe homeostasis.

\section{Methods}

\section{Plant Materials}

The rice cultivar Nipponbare was used in this study as a wild-type control. To construct a binary vector for the over-expression of OsIRO3, the full length cDNA sequence of the OsIRO3 gene was amplified using the following primers:

Forward 5' - GGATCCGATTTGAGCAGGGAACGGAAGG - $3^{\prime}$

Reverse 5'- GGATCCGACAGAAGTGTTTTCGTG TGGA - $3^{\prime}$.

The PCR fragment was then cloned into the Takara pMD19 vector. After sequence confirmation, it was cloned into pTF101-ubi at the BamHI site (Additional file 5) [13]. Agrobacterium-mediated rice transformation of rice callus was carried out as described previously [35]. Ten independently transformed plant lines were generated. QRT-PCR analysis was performed to evaluate the effect of over-expression. Two independent lines were selected with significant increased levels of OsIRO3 transcripts, designated as OE-1 and OE-2.

\section{Plant cultivation conditions}

Wild-type and transgenic plants were germinated and grown in culture solution prepared as described by Yoshida et al [36] except using EDTA-Fe instead of Citrate-Fe. The solution contained $1.425 \mathrm{mM} \mathrm{NH}_{4} \mathrm{NO}_{3}$ , $0.323 \mathrm{mM} \mathrm{NaH} \mathrm{PO}_{4}, 0.513 \mathrm{mM} \mathrm{K} \mathrm{SO}_{4}, 0.998 \mathrm{mM}$ $\mathrm{CaCl}_{2}, 1.643 \mathrm{mM} \mathrm{MgSO}+, 0.009 \mathrm{mM} \mathrm{MnCl}_{2}, 0.075$ $\mu \mathrm{M}\left(\mathrm{NH}_{4}\right)_{6} \mathrm{Mo}_{7} \mathrm{O}_{24}, 0.019 \mathrm{mM} \mathrm{H}_{3} \mathrm{BO}_{3}, 0.155 \mu \mathrm{M}$ $\mathrm{CuSO}_{4}, 0.125 \mathrm{mM}$ FeEDTA and $0.152 \mu \mathrm{M} \mathrm{ZnSO} 4$.

Rice seeds were germinated in distilled water for 2 days. After germination, 25 seedlings were transferred to a plastic net floating on the nutrient solution described above. For metal deficient treatments, two normally growth rice seedlings were transferred to K-, Fe-, Zn-, $\mathrm{Cu}$ - and $\mathrm{Mn}$-free solution. Solution culture was changed every two days. After ten days of metal depletion treatments, root and leaf samples were harvested for RNA extraction and gene expression analysis. For Fe-deficient time-course analysis, rice seedlings were transferred to $+\mathrm{Fe}$ and $-\mathrm{Fe}$ solutions, root and shoot samples for RNA extraction were taken after 1, 3, 6 and 8 days,

For different $\mathrm{Fe}$ concentration treatments, three week old transgenic and wild-type rice plants were transferred to solution culture containing with 100,10 or $1 \mu \mathrm{M}$ EDTA-Fe. After 8 days of treatments, roots and leaves were sampled for RNA extraction, measurements of metal concentration, SPAD and shoot length measurements.

\section{Measurement of chlorophyll content}

SPAD values (total chlorophyll content) were determined on the fully expanded youngest leaves of seedlings with a portable chlorophyll meter (SPAD-502; Minolta Sensing).

\section{Measurement of the Fe concentrations}

To determine the concentrations of Fe in the rice plants, elemental analysis was conducted on seedlings grown under all treatments. Shoot and root samples were ground to a fine powder and digested with $5 \mathrm{~mL}$ of $11 \mathrm{M}$ $\mathrm{HNO}_{3}$ for $5 \mathrm{~h}$ at $150^{\circ} \mathrm{C}$. Metal concentrations were measured using Inductively Coupled Plasma Mass Spectrometry (ICP-MS, Agilent 7500ce, Santa Clara, CA, US).

\section{Quantitative RT-PCR}

Total RNA was extracted from plant samples using TRIzol Reagent (Invitrogen, CA, USA) according to the manufacturer's recommendations. First-strand cDNAs were synthesized from total RNA using SuperScript II reverse 
transcriptase (Invitrogen, CA, USA). QRT-PCR was performed using SYBR Premix Ex Taq ${ }^{\text {mix }}$ (Perfect Real Time) Kit (TaKaRa Biomedicals, Tokyo, Japan) on a LightCycler480 machine (Roche Diagnostics, Basel, Switzerland), according to the manufacturer's instructions. The amplification program for SYBR Green I was performed at $94^{\circ}$ $\mathrm{C}$ for $10 \mathrm{~s}, 58^{\circ} \mathrm{C}$ for $10 \mathrm{~s}$, and $72^{\circ} \mathrm{C}$ for $10 \mathrm{~s}$. Triplicate quantitative assays were performed on each cDNA sample. The housekeeping gene Actin was used as an internal control. The relative level of expression was calculated using the formula $2-\Delta(\Delta C \mathrm{p})$. All the primers that were used for the QRT-PCR are given in Additional file 6.

\section{Phylogenetic analysis}

Alignment and phylogenetic tree were conducted using default settings and the neighbor-joining algorithm of the MEGA version 4 [37] with 1000 bootstrap trials. Amino acid sequences of the bHLH TFs used for the analysis are listed in Additional file 1. Amino acid sequence similarity analysis was conducted in a website tool found at: http://www.ebi.ac.uk/Tools/emboss/align/ index.html.

\section{Statistical analysis of data}

For comparisons of treatments in Figure 4, a two sample t-test assuming unequal variances was performed with all samples compared to wild-type grown in three different levels of Fe supply. Significance was defined as $\mathrm{p} \leq=0.05$.

\section{Additional material}

Additional file 1: The amino acid sequences of bHLH transcription factors used for sequence similarity analysis and phylogenetic tree construction in Figure 3.

Additional file 2: Growth performance of the OsIRO3 overexpression lines and wildtype plant.

Additional file 3: OsIRO2 cis element "G-box plus G" in the promoter region of OsIRO3.

Additional file 4: Expression of OsIRO3 (A) and IDEF1 (B) in different tissues and treatments

Additional file 5: Constructs of $\mathrm{OsIRO3}$ over-expression binary vector.

Additional file 6: Primer sequences used in QRT-PCR analysis

\section{Abbreviations}

bHLH: basic helix loop helix; FRO: ferric reductase/oxidase; IRT: iron regulated transporter; NA: nicotianamine; PS: phytosiderophore; YS: yellow stripe

\section{Acknowledgements}

This work was supported by National Natural Science Foundation of China (30770191 and 30871585) and Harvestplus-China project.

\section{Author details}

'Joint Research Laboratory in Genomics and Nutriomics, College of Life Sciences, Zhejiang University, Hangzhou 310058, P R China. ${ }^{2}$ State Key
Laboratory of Plant Physiology and Biochemistry, College of Life Sciences, Zhejiang University, Hangzhou, 310058, P R China. ${ }^{3}$ Australian Research Council Centre of Excellence in Plant Energy Biology, University of Western Australia, Crawley 6009, WA, Australia.

\section{Authors' contributions}

$L Z, Y Y$ and LW did the physiological, gene expression analysis and transgenic work. LW and FW did the GFP fusion protein and subcellular localization work. LZ and HS participated in the design and coordination of the study. LZ, JW and HS wrote the manuscript. All authors read and approved the final manuscript.

Received: 8 May 2010 Accepted: 11 August 2010

Published: 11 August 2010

\section{References}

1. Buchanan B, Gruissem W, Jones R: Biochemistry and Molecular Biology of Plants. John Wiley \& Sons Inc, Somerset, NJ, USA 2000.

2. Guerinot ML, Yi Y: Iron: nutritious, noxious, and not readily available. Plant Physiol 1994, 104(3):815-820

3. Kim SA, Guerinot ML: Mining iron: iron uptake and transport in plants. FEBS letters 2007, 581(12):2273-2280

4. Marschner $H$, Römheld V, Kissel M: Different strategies in higher plants in mobilization and uptake of iron. J Plant Nutr 1986, 9:695-713.

5. Eide D, Broderius M, Fett J, Guerinot ML: A novel iron-regulated metal transporter from plants identified by functional expression in yeast. Proc Natl Acad Sci USA 1996, 93:5624-5628.

6. Robinson NJ, Procter CM, Connolly EL, Guerinot ML: A ferric-chelate reductase for iron uptake from soils. Nature 1999, 397(6721):694-697.

7. Curie C, Panaviene Z, Loulergue C, Dellaporta SL, Briat JF, Walker EL: Maize yellow stripe1 encodes a membrane protein directly involved in $\mathrm{Fe}(\mathrm{III})$ uptake. Nature 2001, 409(6818):346-349.

8. Inoue H, Kobayashi T, Nozoye T, Takahashi M, Kakei Y, Suzuki K, Nakazono M, Nakanishi H, Mori S, Nishizawa NK: Rice OsYSL15 Is an Ironregulated Iron(III)-Deoxymugineic Acid Transporter Expressed in the Roots and Is Essential for Iron Uptake in Early Growth of the Seedlings. J Biol Chem 2009, 284:10.

9. Murata Y, Ma JF, Yamaji N, Ueno D, Nomoto K, Iwashita T: A specific transporter for iron(III)-phytosiderophore in barley roots. Plant J 2006, 46(4):563-572.

10. Santi S, Schmidt W: Dissecting iron deficiency-induced proton extrusion in Arabidopsis roots. New Phytologist 2009, 183(4):1072-1084.

11. Curie C, Briat JF: Iron transport and signaling in plants. Annu Rev Plant Biol 2003, 54(1):183-206.

12. Inoue H, Higuchi K, Takahashi M, Nakanishi H, Mori S, Nishizawa NK: Three rice nicotianamine synthase genes, OsNAS1, OsNAS2, and OsNAS3 are expressed in cells involved in long-distance transport of iron and differentially regulated by iron. Plant J 2003, 36(3):366-381.

13. Cheng L, Wang F, Shou H, Huang F, Zheng L, He F, Li J, Zhao FJ, Ueno D, $\mathrm{Ma} J \mathrm{~J}$, et al: Mutation in nicotianamine aminotransferase stimulated the $\mathrm{Fe}$ (II) acquisition system and led to iron accumulation in rice. Plant Physiol 2007, 145(4):1647-1657.

14. Walker EL, Connolly EL: Time to pump iron: iron-deficiency-signaling mechanisms of higher plants. Curr Opin Plant Biol 2008, 11(5):530-535.

15. Ogo Y, Itai RN, Nakanishi H, Inoue H, Kobayashi T, Suzuki M, Takahashi M, Mori S, Nishizawa NK: Isolation and characterization of IRO2, a novel ironregulated bHLH transcription factor in graminaceous plants. Journal of experimental botany 2006, 57(11):2867-2878.

16. Thimm O, Essigmann B, Kloska S, Altmann T, Buckhout TJ: Response of Arabidopsis to iron deficiency stress as revealed by microarray analysis. Plant Physiol 2001, 127(3):1030-1043.

17. Zheng L, Huang F, Narsai R, Wu J, Giraud E, He F, Cheng L, Wang F, Wu P, Whelan J, et al: Physiological and Transcriptome Analysis of Iron and Phosphorus Interaction in Rice Seedlings. Plant Physiol 2009, 151(1):262-274.

18. Ling HQ, Bauer P, Bereczky Z, Keller B, Ganal M: The tomato fer gene encoding a bHLH protein controls iron-uptake responses in roots. Proc Natl Acad Sci USA 2002, 99(21):13938-13943.

19. Colangelo EP, Guerinot ML: The essential basic helix-loop-helix protein FIT1 is required for the iron deficiency response. Plant Cell 2004, 16:3400-3412. 
20. Wang $H$, Klatte $M$, Jakoby $M$, Bäumlein $H$, Weisshaar B, Bauer P: Iron deficiency-mediated stress regulation of four subgroup $\mathrm{lb}$ BHLH genes in Arabidopsis thaliana. Planta 2007, 226:897-908.

21. Yuan Y, Wu H, Wang N, Li J, Zhao W, Du J, Wang D, Ling HQ: FIT interacts with AtbHLH38 and AtbHLH39 in regulating iron uptake gene expression for iron homeostasis in Arabidopsis. Cell Res 2008, 18(3):385-397.

22. Ogo Y, Itai RN, Nakanishi H, Kobayashi T, Takahashi M, Mori S, Nishizawa NK: The rice bHLH protein OsIRO2 is an essential regulator of the genes involved in Fe uptake under Fe-deficient conditions. Plant J 2007, 51(3):366-377.

23. Kobayashi T, Nakayama Y, Itai RN, Nakanishi H, Yoshihara T, Mori S, Nishizawa NK: Identification of novel cis-acting elements, IDE1 and IDE2, of the barley IDS2 gene promoter conferring iron-deficiency-inducible, root-specific expression in heterogeneous tobacco plants. Plant J 2003, 36(6):780-793.

24. Kobayashi T, Ogo Y, Itai RN, Nakanishi H, Takahashi M, Mori S, Nishizawa NK: The transcription factor IDEF1 regulates the response to and tolerance of iron deficiency in plants. Proc Natl Acad Sci USA 2007, 104(48):19150-19155.

25. Ogo Y, Kobayashi T, Nakanishi Itai R, Nakanishi H, Kakei Y, Takahashi M, Toki S, Mori S, Nishizawa N: A novel NAC transcription factor, IDEF2, that recognizes the iron deficiency-responsive element 2 regulates the genes involved in iron homeostasis in plants. J Biol Chem 2008, 283:13407-13417.

26. Li X, Duan X, Jiang H, Sun Y, Tang Y, Yuan Z, Guo J, Liang W, Chen L, Yin J, et al: Genome-wide analysis of basic/helix-loop-helix transcription factor family in rice and Arabidopsis. Plant Physiol 2006, 141(4):1167-1184.

27. Pires N, Dolan L: Origin and Diversification of Basic-Helix-Loop-Helix Proteins in Plants. Mol Biol Evol 2010, 27(4):862-874.

28. Rampey RA, Woodward AW, Hobbs BN, Tierney MP, Lahner B, Salt DE, Bartel B: An Arabidopsis basic helix-loop-helix leucine zipper protein modulates metal homeostasis and auxin conjugate responsiveness. Genetics 2006, 174(4):1841-1857.

29. Wang YJ, Zhang ZG, He XJ, Zhou HL, Wen YX, Dai JX, Zhang JS, Chen SY: A rice transcription factor OsbHLH1 is involved in cold stress response. Theor Appl Genet 2003, 107(8):1402-1409.

30. Belouchi A, Kwan T, Gros P: Cloning and characterization of the OsNramp family from Oryza sativa, a new family of membrane proteins possibly implicated in the transport of metal ions. Plant Molecular Biology 1997, 33(6):1085-1092

31. Cassin G, Mari S, Curie C, Briat JF, Czernic P: Increased sensitivity to iron deficiency in Arabidopsis thaliana overaccumulating nicotianamine. Journal of experimental botany 2009, 60:1249-1259.

32. Zheng L, Cheng Z, Ai C, Jiang X, Bei X, Zheng Y, Glahn RP, Welch RM, Miller DD, Lei $X G$, et al: Nicotianamine, a Novel Enhancer of Rice Iron Bioavailability to Humans. PLOS ONE 2010, 5(4):10190.

33. Ma P, Rould M, Weintraub H, Pabo C: Crystal structure of MyoD bHLH domain-DNA complex: perspectives on DNA recognition and implications for transcriptional activation. Cell 1994, 77:451-459.

34. Shimizu T, Toumoto A, Ihara K, Shimizu M, Kyogoku Y, Ogawa N, Oshima Y, Hakoshima T: Crystal structure of PHO4 bHLH domain-DNA complex: flanking base recognition. EMBO J 1997, 16:4689-4697.

35. Chen $S$, Jin W, Wang M, Zhang F, Zhou J, Jia Q, Wu Y, Liu F, Wu P: Distribution and characterization of over 1000 T-DNA tags in rice genome. Plant J 2003, 36:105-113.

36. Yoshida S, Forno DA, Cock JH, KA G: Laboratory Manual for Physiological Studies of Rice. The International Rice Research Institute: Manila, The Philippines, 31976.

37. Tamura K, Dudley J, Nei M, S K: MEGA4: Molecular Evolutionary Genetics Analysis (MEGA) software version 4.0. Mol Biol Evol 2007, 24:1596-1599.

\section{doi:10.1186/1471-2229-10-166}

Cite this article as: Zheng, et al:: Identification of a novel iron regulated basic helix-loop-helix protein involved in Fe homeostasis in Oryza sativa. BMC Plant Biology 2010 10:166. 